\section{Diferenças por sexo e idade no preenchimento da escolaridade em fichas de vigilância em capitais brasileiras com maior incidência de dengue, 2008-2017}

\author{
Gender and age differences in the completion \\ of the schooling items in surveillance forms \\ in Brazilian state capitals with higher dengue \\ incidence, 2008-2017
}

Diferencias por sexo y edad para la cumplimentación de la escolaridad en fichas de vigilancia en las capitales brasileñas con mayor incidencia de dengue, 2008-2017

\section{Resumo}

O Brasil figura entre os países com o maior número de casos de dengue no mundo. Sistemas de notificação são fundamentais para acompanhar a evolução do agravo, porém são pouco preenchidos. O conhecimento do nível educacional dos indivíduos é importante para o entendimento do papel da desigualdade social no risco de adoecimento. O objetivo deste artigo é descrever o preenchimento da escolaridade nas fichas de notificação para dengue disponibilizadas pelo Sistema de Informação de Agravos de Notificação (SINAN) entre 2008 e 2017, por sexo e faixa etária, entre as capitais das regiões Nordeste e Sudeste do país. O desfecho de interesse foi o preenchimento da variável escolaridade. As probabilidades preditas de preenchimento da escolaridade foram obtidas utilizando-se modelos logísticos multiníveis. Esse preenchimento foi baixo nas capitais, sendo em oito delas inferior a $30 \%$ no período. A chance de ter a escolaridade preenchida decresceu com o avançar da idade, independentemente do sexo e do ano. Mulheres com até 60 anos apresentaram maiores chances de preenchimento da escolaridade que os homens. De um modo geral, os homens registraram 9\% menos chances de ter a escolaridade preenchida do que as mulheres. No modelo com interação houve uma intensificação da diferença, ou seja, houve uma piora no preenchimento com o progredir da idade, principalmente entre as mulheres. Diante do exposto, o mau preenchimento das fichas favorece a geração de dados deficientes e duvidosos, e a escolaridade, embora pouco valorizada no ato do preenchimento, tem importância central nesta conjuntura.

Dengue; Sistemas de Informação em Saúde; Escolaridade
Lucas Melo Guimarães 1

Geraldo Marcelo da Cunha 2

doi: 10.1590/0102-311X00187219

Correspondência

L. M. Guimarães

Rua Domingos de Pádua Rego 3700, casa 16, Teresina, PI 64002-595, Brasil.

lucasmeloguimaraes@gmail.com

1 Fundação Municipal de Saúde, Teresina, Brasil. 2 Escola Nacional de Saúde Pública Sergio Arouca, Fundação Oswaldo Cruz, Rio de Janeiro, Brasil. 


\section{Introdução}

Dados do Sistema de Informação de Agravos de Notificação (SINAN) são amplamente utilizados como uma importante ferramenta no monitoramento da incidência e pesquisa da dengue. Os usuários dos dados dependem da integridade e confiabilidade do registro, para que seus achados sejam válidos e possibilitem tomadas de decisão, alocação de recursos, identificação de grupos sujeitos a um maior risco e melhor entendimento da etiologia dos agravos 1. Entretanto, a exemplo do que ocorre nos outros sistemas de informações em saúde no Brasil, o preenchimento de variáveis como a escolaridade é baixo 2 .

O conhecimento do nível educacional dos indivíduos é importante para o entendimento do papel da desigualdade social no risco de adoecimento. Alguns autores consideram a educação como o mais forte preditor socioeconômico individual de boa saúde, e variável mais consistentemente associada a diversos agravos 3,4. O efeito da educação na saúde é multicausal; indivíduos com maior escolaridade usufruem de melhores condições financeiras e sociais na vida adulta, o que pode promover o acesso a alimentos mais saudáveis, influenciar práticas benéficas e contrárias a comportamentos de risco conhecidos, além de apresentar efeitos positivos em aspectos psicológicos como autoestima, estresse e depressão, obtendo, assim, maior satisfação com a vida e melhor saúde 5,6. Quando comparado a outros países do mundo, a renda futura média de um brasileiro dependerá fortemente de sua escolaridade. Ou seja, um maior grau de escolaridade refletirá também no acesso a um melhor serviço de saúde 7 . Nesse sentido, as informações sobre o perfil educacional é essencial para orientar políticas públicas.

As informações coletadas nas fichas de notificação são preenchidas por diversos profissionais do serviço de saúde, de modo que seria esperado semelhanças nas probabilidades de preenchimento da escolaridade por sexo e idade por capital. O objetivo do presente estudo foi estimar o grau de preenchimento da variável escolaridade no SINAN nos casos notificados de dengue, estratificando por sexo, idade e capitais das regiões Nordeste e Sudeste do Brasil no período de 2008 a 2017.

\section{Métodos}

\section{Fonte de dados}

O SINAN é um sistema essencial para as atividades de vigilância epidemiológica, integra o Departamento de Informática do SUS (DATASUS), mantido pelo Ministério da Saúde. Foi fundado no início dos anos 1990, porém sua implantação em todo o território brasileiro ocorreu apenas em 1998 8,9. O registro é baseado na notificação de agravos do dengue e outros agravos como tuberculose, febre amarela e malária. O SINAN monitora a saúde da população, prevê ocorrências de agravos e auxilia no planejamento, definição de prioridades e avaliação do impacto de intervenções realizadas ${ }^{8}$. É mandatório que todos os prestadores de cuidados de saúde relatem os casos suspeitos de dengue ao SINAN. Nesses casos, a ficha de notificação é aberta e, sempre que possível, exames laboratoriais são solicitados para a confirmação, que pode ocorrer também clinicamente. Independentemente da confirmação, o registro é inserido no sistema por intermédio das secretarias municipais e estaduais de saúde 10 .

A informação disponível no SINAN inclui dados do paciente (nome, sexo, idade, escolaridade, local de residência, dentre outros); dados médicos (sintomatologia, doenças preexistentes, dados laboratoriais etc.); e dados de acompanhamento (classificação, confirmação, evolução, datas de óbito/ alta e encerramento).

Considerou-se inicialmente todos os casos notificados para dengue disponibilizados pelo SINAN nas capitais das regiões Nordeste e Sudeste do Brasil (São Luís, Teresina, Fortaleza, Natal, João Pessoa, Recife, Maceió, Aracaju, Salvador, Belo Horizonte, Vitória, Rio de Janeiro e São Paulo), entre os anos de 2008 e 2017.

As capitais das regiões Nordeste e Sudeste apresentam uma melhor estrutura logística de pessoal e serviços, maior capacidade de adequação às normativas nacionais e balizam as ações dos municípios de menor porte dentro de seus respectivos estados 11. As capitais também concentram uma maior 
quantidade de casos notificados em relação ao restante do estado. Por exemplo, 43,7\% dos casos notificados de dengue no Estado do Ceará em 2016 referiam-se a residentes na capital Fortaleza.

Foram excluídos da população de casos notificados os pacientes menores de 20 anos, uma vez que o nível educacional é obtido pelas pessoas, em geral, durante o período que vai da infância até a idade adulta jovem. Eliminou-se também os pacientes com idade informada maior ou igual a 99 anos. É possível que uma parte desses dados tenha sido preenchida de forma equivocada. Por exemplo, um número moderadamente elevado de pacientes com 99 anos mostra indícios desse erro no preenchimento. Também foram excluídos os paciente com preenchimento "Ignorado" para o sexo e "Não se aplica" para a escolaridade.

O desfecho de interesse foi o preenchimento ou não da variável escolaridade. A escolaridade foi classificada como não preenchida caso seu preenchimento original fosse "Ignorado" ou ausente, e classificada como preenchida para as demais opções de resposta. Foram consideradas para as análises as variáveis sexo, idade, município de residência e ano de ocorrência.

\section{Métodos estatísticos}

A fim de avaliar diferenças no preenchimento da escolaridade por sexo e idade, as probabilidades de preenchimento da variável e seus respectivos intervalos de 95\% de confiança (IC95\%) foram calculados estratificando-se sob diferentes combinações por sexo, idade, ano de ocorrência e capital.

As probabilidades do preenchimento da variável escolaridade por capital são bastante discrepantes (Tabela 1). Desse modo, modelos logísticos multiníveis foram usados com a finalidade de levar em conta a correlação na chance de preenchimento da escolaridade para residentes de uma mesma capital. Foram considerados dois níveis hierárquicos, nos quais os indivíduos notificados corresponderam às unidades do primeiro nível e as capitais às unidades do segundo nível. Mais especificamente, se $y_{i j}$ indica o preenchimento ou não da escolaridade do indivíduo $i$ residente na capital $j$, assumimos que $y_{i j} \sim \operatorname{Bernoulli}\left(\pi_{i j}\right)$ e temos a seguinte equação:

$$
\left.\log \left(\frac{\pi_{i j}}{1-\pi_{i j}}\right)=\beta_{0}+u_{j}+\beta_{1} \text { sexo }_{i j}+\beta_{2} \text { idade (40-60 anos }\right)_{i j}+\beta_{2} \text { idade }(>60 \text { anos })_{i j}
$$

na qual $\pi_{i j}$ é a probabilidade de preenchimento da escolaridade e $u_{j} \sim N\left(0, \sigma_{u}^{2}\right)$ o termo aleatório.

Considerando conjuntamente os dados de todos os anos, foram ajustados modelos com e sem interação das variáveis sexo e idade de modo a medir o efeito geral destas variáveis. Posteriormente, um modelo com interação foi ajustado para cada ano isoladamente, de modo a obter as razões de chance preditas por sexo e idade ao longo da série histórica. $O$ teste da razão da máxima verossimilhança no nível de significância de 5\% foi usado quando necessário para comparação e avaliação da significância de parâmetros dos modelos.

As análises foram realizadas utilizando-se as bibliotecas foreign, lubridate, ggplot2, lattice, plyr, Rmisc, lme 4 e lsmeans do software R, versão 3.4.4 (http://www.r-project.org).

$\mathrm{O}$ artigo seguiu os preceitos da ética em pesquisa, trabalhando com dados não identificados, disponibilizados a qualquer cidadão pelo Sistema Eletrônico do Serviço de Informação ao Cidadão (https://esic.cgu.gov.br/sistema/site/index.aspx).

\section{Resultados}

Após a aplicação dos critérios de exclusão, foram mantidos aproximadamente 972 mil casos notificados de dengue no período 2008-2017 nas 13 capitais do estudo. Houve predominância do sexo feminino $(57,6 \%)$ e idade de $20-40$ anos $(57,7 \%)$. Entre as capitais, Belo Horizonte (28\%) se destaca como a capital com o maior número de casos e São Luís $(1,1 \%)$ com o menor. Na série de anos analisada, as maiores ocorrências foram registradas em 2016 (17,9\%) e as menores em 2009 (3,2\%).

O preenchimento da variável escolaridade foi baixo nas capitais, tendo oito delas apresentado número inferior a $30 \%$ no período total do estudo. A probabilidade de preenchimento médio variou bastante entre as capitais, já que relativamente houve menor variabilidade nas probabilidades dentro de uma mesma capital ao longo da série histórica analisada. São Luís na Região Nordeste e Vitória na 
Tabela 1

Probabilidade de preenchimento da variável escolaridade na ficha de dengue do Sistema de Informação de Agravos de Notificação (SINAN) por capitais brasileiras das regiões Nordeste e Sudeste, 2008-2017.

\begin{tabular}{llllllllllll}
\hline Capital & $\mathbf{2 0 0 8}$ & $\mathbf{2 0 0 9}$ & $\mathbf{2 0 1 0}$ & $\mathbf{2 0 1 1}$ & $\mathbf{2 0 1 2}$ & $\mathbf{2 0 1 3}$ & $\mathbf{2 0 1 4}$ & $\mathbf{2 0 1 5}$ & $\mathbf{2 0 1 6}$ & $\mathbf{2 0 1 7}$ & Total \\
\hline São Luís & 0,918 & 0,890 & 0,775 & 0,710 & 0,825 & 0,759 & 0,647 & 0,740 & 0,753 & 0,849 & 0,761 \\
Teresina & 0,674 & 0,807 & 0,878 & 0,864 & 0,696 & 0,418 & 0,473 & 0,491 & 0,340 & 0,331 & 0,582 \\
Fortaleza & 0,164 & 0,158 & 0,194 & 0,334 & 0,389 & 0,447 & 0,439 & 0,341 & 0,158 & 0,121 & 0,266 \\
Natal & 0,105 & 0,051 & 0,077 & 0,176 & 0,271 & 0,202 & 0,166 & 0,294 & 0,157 & 0,057 & 0,175 \\
João Pessoa & 0,446 & 0,473 & 0,365 & 0,239 & 0,270 & 0,294 & 0,255 & 0,328 & 0,201 & 0,168 & 0,263 \\
Recife & 0,145 & 0,141 & 0,106 & 0,090 & 0,083 & 0,076 & 0,067 & 0,063 & 0,057 & 0,060 & 0,078 \\
Maceió & 0,178 & 0,110 & 0,138 & 0,328 & 0,311 & 0,417 & 0,264 & 0,280 & 0,247 & 0,322 & 0,236 \\
Aracaju & 0,411 & 0,371 & 0,588 & 0,373 & 0,306 & 0,129 & 0,092 & 0,137 & 0,075 & 0,512 & 0,292 \\
Salvador & 0,121 & 0,175 & 0,219 & 0,266 & 0,273 & 0,152 & 0,138 & 0,080 & 0,074 & 0,116 & 0,170 \\
Vitória & 0,581 & 0,529 & 0,597 & 0,587 & 0,644 & 0,793 & 0,668 & 0,672 & 0,714 & 0,665 & 0,674 \\
Belo Horizonte & 0,588 & 0,504 & 0,500 & 0,444 & 0,449 & 0,440 & 0,391 & 0,304 & 0,212 & 0,242 & 0,358 \\
Rio de Janeiro & 0,112 & 0,121 & 0,192 & 0,047 & 0,138 & 0,111 & 0,175 & 0,223 & 0,262 & 0,259 & 0,131 \\
São Paulo & 0,510 & 0,558 & 0,477 & 0,538 & 0,600 & 0,558 & 0,514 & 0,477 & 0,434 & 0,552 & 0,504 \\
Total & 0,221 & 0,390 & 0,398 & 0,266 & 0,234 & 0,377 & 0,418 & 0,333 & 0,217 & 0,216 & 0,195 \\
\hline
\end{tabular}

Região Sudeste foram as capitais que apresentaram os melhores preenchimentos. As capitais que apresentaram os piores registros de escolaridade em cada região foram Recife e Rio de Janeiro (Tabela 1).

A chance de ter a escolaridade preenchida decresceu gradativamente com o avançar da idade e alcançou os piores valores em pacientes com 70 anos ou mais, independentemente do sexo e do ano. De um modo geral, mulheres com até 60 anos de idade apresentaram as maiores chances de preenchimento da escolaridade que os homens. Entre os idosos de 70 anos ou mais, os preenchimentos foram em geral maiores dentre os homens (Figura 1). Optamos por não utilizar a mesma escala para o preenchimento da escolaridade ao longo dos anos, para destacar os efeitos das variáveis sexo e idade.

O padrão temporal nas chances de preenchimento da escolaridade entre capitais foi diverso. Em João Pessoa, Recife, Teresina e Belo Horizonte houve um decréscimo nas probabilidades de preenchimento ao longo dos anos. Inversamente, houve uma melhora crescente na chance de ter a escolaridade preenchida em Vitória, Rio de Janeiro e Maceió. Outras capitais apresentaram padrões de melhora e piora no preenchimento da variável ao longo da série. Em geral, as mulheres obtiveram maiores chances de preenchimento independentemente do ano e da capital considerados no estudo, mas sobretudo nas capitais com as maiores ocorrências: Belo Horizonte, Rio de Janeiro e São Paulo (Figura 2).

De um modo geral, a chance de um homem ter escolaridade preenchida foi $9 \%$ menor que uma mulher. A chance de ter a escolaridade preenchida decresce com a idade, sendo $25 \%$ menor nos maiores de 60 anos em comparação com aqueles com idades entre 20 e 40 anos (Modelo 1, Tabela 2). No modelo com interação (Modelo 2, Tabela 2), houve uma intensificação dessa diferença para as mulheres (chance 27\% menor nas maiores de 60 anos comparadas às de 20-40) e menor diferença entre os homens (chance de $21 \%$ menor nos maiores de 60 anos comparados aos de 20-40), ou seja, houve uma piora no preenchimento da escolaridade com o progredir da idade, principalmente entre as mulheres. Independente do modelo, com ou sem interação, $24 \%$ da variação residual na probabilidade de ter a variável escolaridade preenchida foram atribuídas a características não observadas nas capitais.

Após os ajustes dos modelos com interação ano a ano, a chance de ter a escolaridade preenchida foi mais próxima em pacientes com idades de 20-40 anos e 40-60 anos, principalmente entre as mulheres. Tomando os anos de maior incidência (2015 e 2016), as diferenças de preenchimentos entre mulheres e homens foram as mais acentuadas no comparativo entre idades mais jovens e os maiores de 60 anos. Particularmente em 2017, houve melhor preenchimento entre os homens na idade entre 40-60 anos quando comparados aos de 20-40 anos. Contudo, independentemente do ano, as discrepâncias maiores nas razões de chance ocorreram entre as mulheres idosas quando comparadas às mais jovens, fato que também ocorreu entre os homens, mas em menor magnitude (Figura 3). 


\section{Figura 1}

Probabilidade de preenchimento da variável escolaridade na ficha de dengue do Sistema de Informação de Agravos de Notificação (SINAN) nas capitais brasileiras das regiões Nordeste e Sudeste, por idade e sexo, estratificado por ano de ocorrência entre 2008 e 2017.

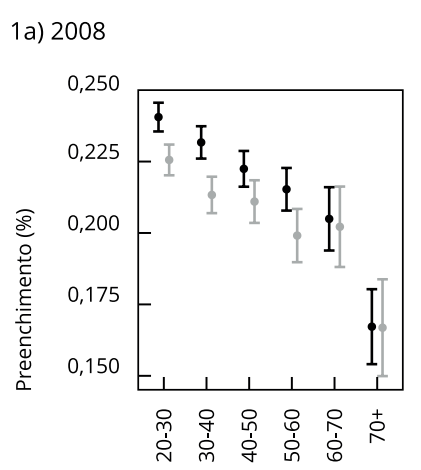

Faixa etária (anos)

1e) 2012

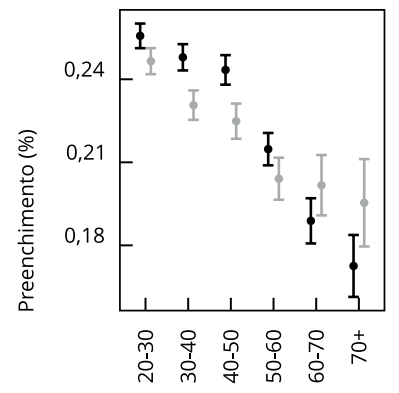

Faixa etária (anos)

1i) 2016

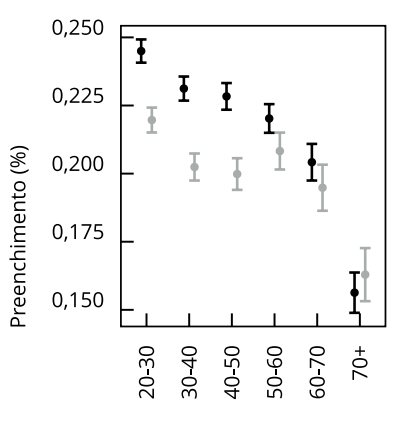

Faixa etária (anos) 1b) 2009

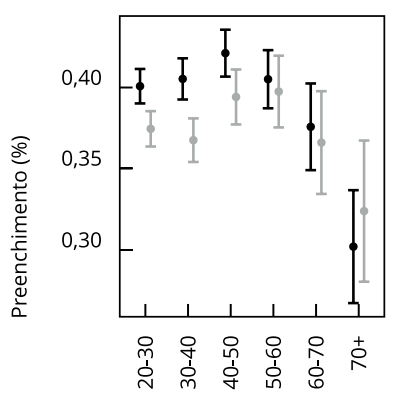

Faixa etária (anos)

1f) 2013

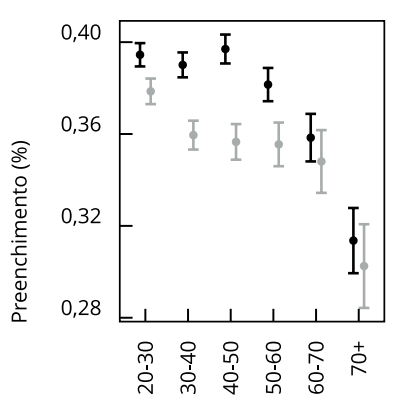

Faixa etária (anos) 1c) 2010

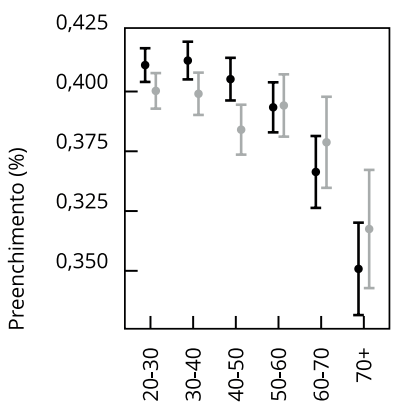

Faixa etária (anos)

1g) 2014

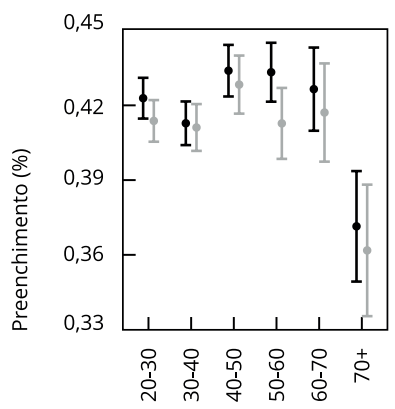

Faixa etária (anos) 1d) 2011

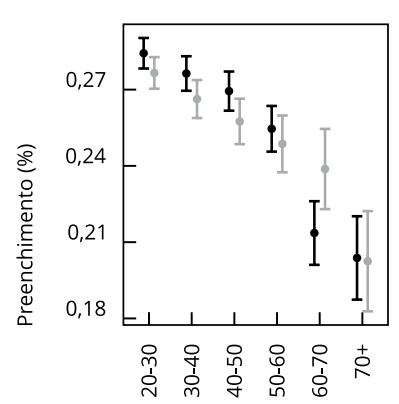

Faixa etária (anos)

1h) 2015

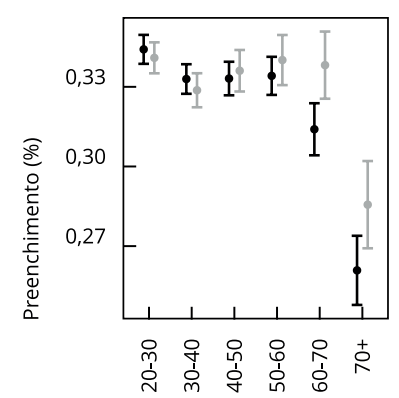

Faixa etária (anos)

1j) 2017

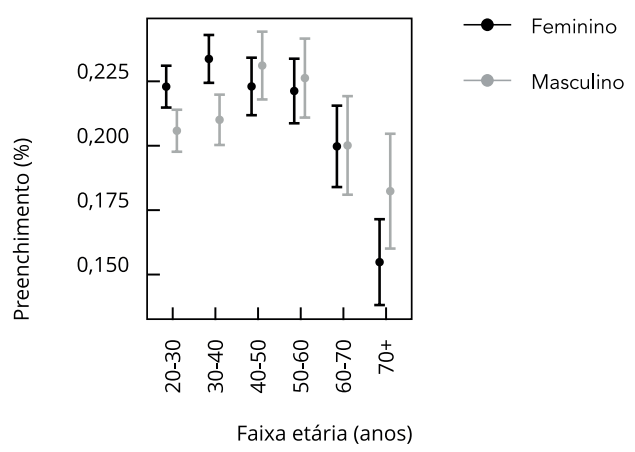


Figura 2

Probabilidade de preenchimento da variável escolaridade na ficha de dengue do Sistema de Informação de Agravos de Notificação (SINAN), por ano de ocorrência e sexo, estratificado pelas capitais brasileiras das regiões Nordeste e Sudeste entre 2008 e 2017.

2a) São Luís

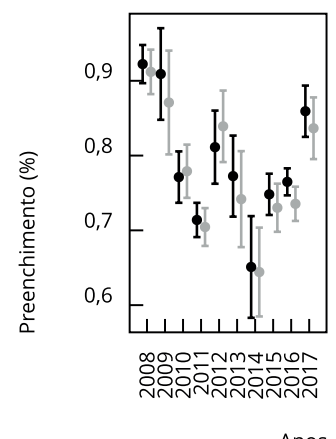

2f) Recife

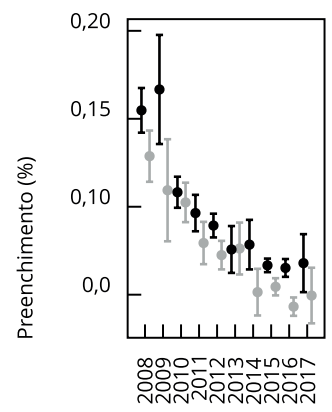

Anos 2b) Teresina

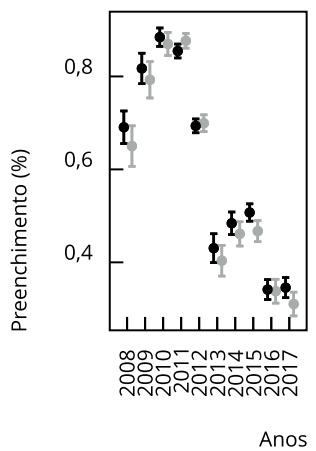

2g) Maceió

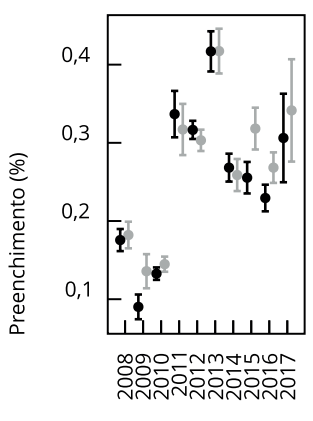

Anos 2c) Fortaleza

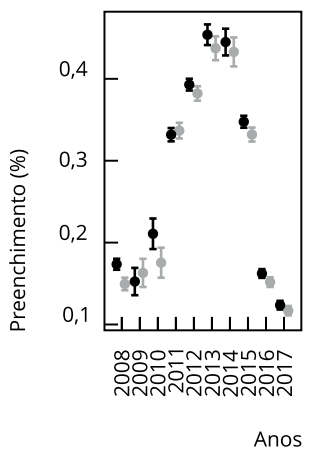

2h) Aracaju

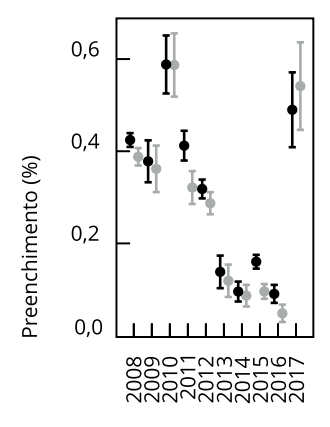

Anos 2d) Natal

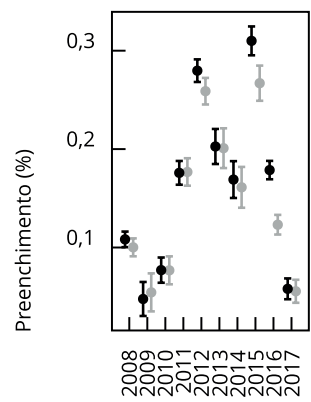

Anos

2i) Salvador

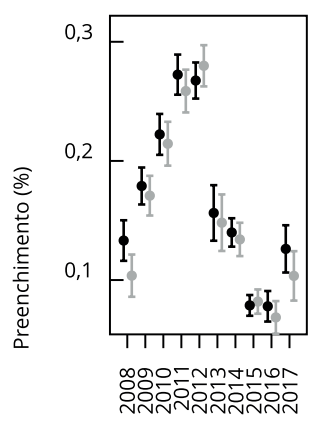

Anos 2e) João Pessoa

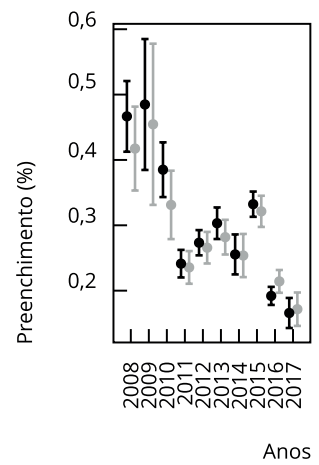

2j) Vitória

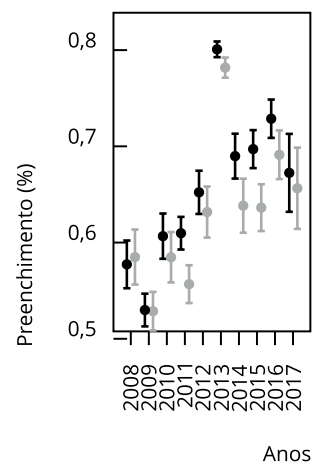

2k) Belo Horizonte

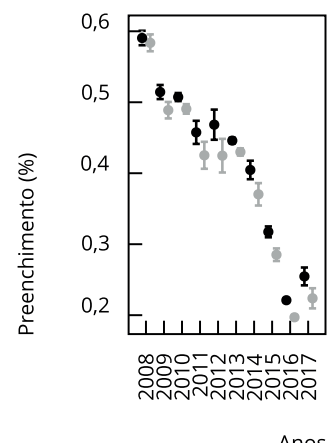

2l) Rio de Janeiro

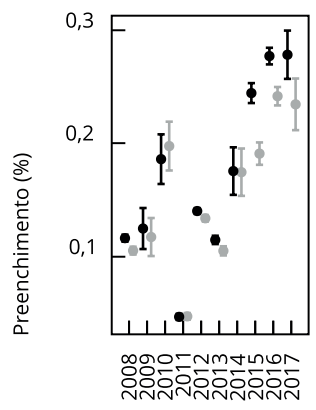

Anos 2m) São Paulo

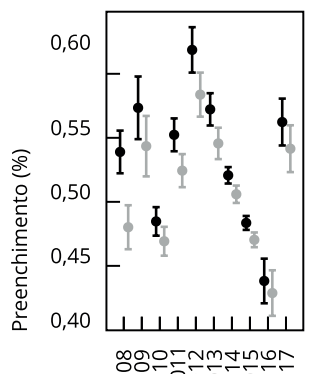

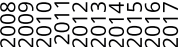

Anos $\longrightarrow$ Feminino
$\longrightarrow \quad$ Masculino 
Tabela 2

Modelo estatístico multinível da relação entre preenchimento da variável escolaridade por sexo e idade, nas capitais brasileiras das regiões Nordeste e Sudeste, 2008-2017.

\begin{tabular}{|c|c|c|c|c|}
\hline \multirow[t]{2}{*}{ Variáveis * } & \multicolumn{2}{|c|}{ Modelo 1} & \multicolumn{2}{|c|}{ Modelo 2} \\
\hline & OR (IC95\%) & Valor de p & OR (IC95\%) & Valor de $p$ \\
\hline \multicolumn{5}{|l|}{ Sexo } \\
\hline Masculino & $0,91(0,90-0,92)$ & $<0,001$ & $0,91(0,90-0,92)$ & $<0,001$ \\
\hline \multicolumn{5}{|l|}{ Idade (anos) } \\
\hline $40-60$ & $0,95(0,94-0,96)$ & $<0,001$ & $0,96(0,95-0,97)$ & $<0,01$ \\
\hline$>60$ & $0,75(0,74-0,76)$ & $<0,001$ & $0,73(0,710,74)$ & $<0,01$ \\
\hline \multicolumn{5}{|l|}{ Interações } \\
\hline Masculino | 40-60 anos & - & - & $0,98(0,94-0,99)$ & 0,03 \\
\hline Masculino | > 60 anos & - & - & $1,08(1,06-1,10)$ & $<0,01$ \\
\hline Coeficiente de partição da variância & \multicolumn{2}{|c|}{0,24} & \multicolumn{2}{|c|}{0,24} \\
\hline
\end{tabular}

IC95\%: intervalo de 95\% de confiança; OR: razão de chances.

* Os valores de referência para as variáveis foram: Feminino para o sexo e 20-40 anos para a idade.

\section{Discussão}

O preenchimento da variável escolaridade foi baixo $(19,5 \%)$ nas fichas de notificação de dengue entre as capitais das regiões Nordeste e Sudeste do Brasil entre 2008 e 2017. O preenchimento foi melhor para mulheres e diminuiu com o avançar da idade em ambos os sexos, sendo maior para as mulheres com idade menor que 60 anos e para os homens com idade maior que 70 anos.

A falta de capacitação dos profissionais, autoridades responsáveis pelo controle com outras prioridades, desconhecimento da importância das informações coletadas e inadequações no número de recursos humanos e infraestrutura são alguns dos motivos apontados como razões do não preenchimento de variáveis em fichas de sistemas de informação em saúde, notadamente aqui a escolaridade $8,12,13,14$. Muitos desses motivos são intrínsecos a modelos de gestão de cada capital. Considerando que esses modelos se perpetuam por períodos prolongados (minimamente o mandato de 4 anos de cada prefeito), são esperadas tendências de preenchimentos crescentes ou decrescentes entre as capitais ou mesmo discrepâncias, rupturas mais acentuadas entre governos. Teresina, por exemplo, passou por mudanças administrativas severas na saúde em 2013, com a separação de sua estrutura que outrora era centralizada em Fundação Municipal de Saúde em outros três órgãos 15. Porém, o pretexto de melhor organizar processos teve um resultado reverso, como podemos observar na queda de preenchimento da escolaridade a partir desse ano para essa capital.

A melhoria na completude das variáveis requer um esforço conjunto desde os gestores até os profissionais responsáveis pelo preenchimento nos estabelecimentos de saúde 11. A diversidade dos profissionais que preenchem as fichas de notificação repercute diretamente na completude das variáveis. Eles podem ser de diferentes classes profissionais e graus de formação, com conhecimento insuficiente da ficha de notificação e dando pouca importância às informações anotadas 16,17,18,19. Essas dificuldades de compreensão podem determinar resultados discrepantes, já que a variável questionada pode não estar clara ao profissional, que não oferecerá esclarecimentos oportunos ao respondente, o qual não responderá ou responderá de forma incorreta.

Nosso estudo mostrou haver uma diferença na chance de preenchimento da escolaridade por sexo e idade do paciente atendido, contrariando a racionalidade de que haveria semelhanças nos valores, uma vez que o preenchimento é realizado por um profissional externo à assistência direta ao paciente. Sabe-se que o nível de escolaridade tem significado que varia ao longo do tempo, com implicações sociais e em saúde se relacionando com idade, coorte de nascimento, posição de classe social, raça e sexo 4,20. Ou seja, para diferentes idades e sexos há acepções diferentes desse indicador, o que por si pode refletir em distintos entendimentos de sua importância ou em seu preenchimento ou não. 
Figura 3

Razões de chance (OR) preditas do preenchimento da escolaridade por sexo e idade, nas capitais das regiões Nordeste e Sudeste do Brasil, $2008-2017$.

3a) 2008

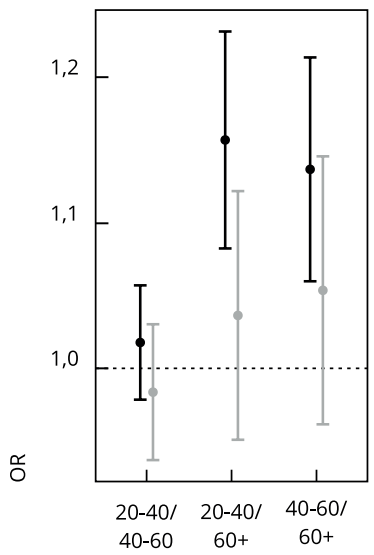

Contrastes [Faixa etária (anos)]

3e) 2012

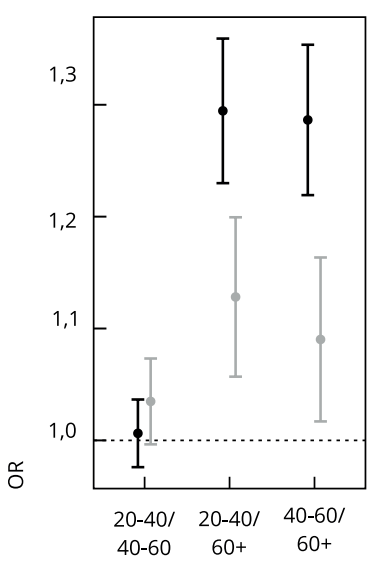

Contrastes [Faixa etária (anos)]

3i) 2016

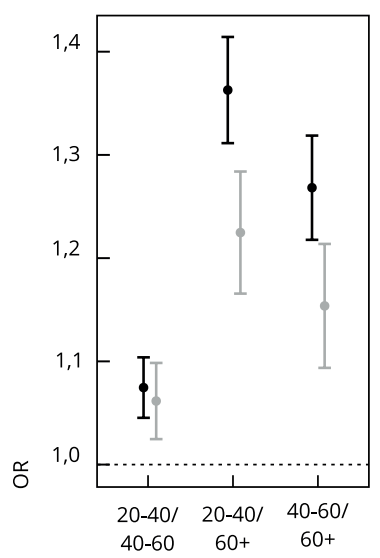

Contrastes [Faixa etária (anos)] 3b) 2009

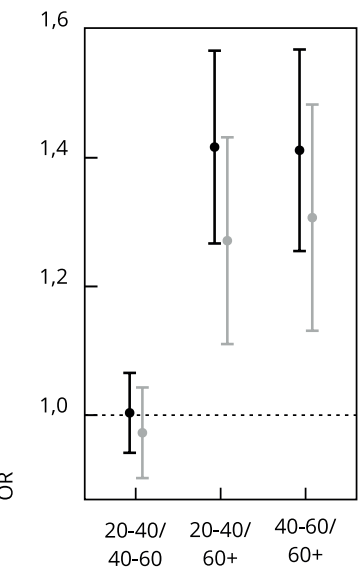

Contrastes [Faixa etária (anos)]

3f) 2013

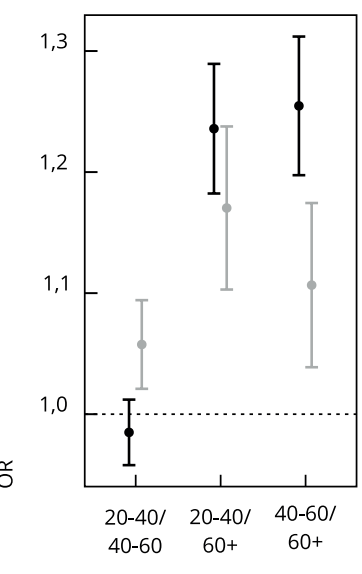

Contrastes [Faixa etária (anos)]

3j) 2017

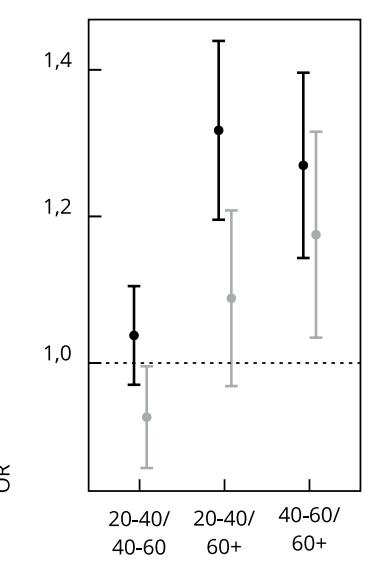

Contrastes [Faixa etária (anos)] 3c) 2010

3d) 2011

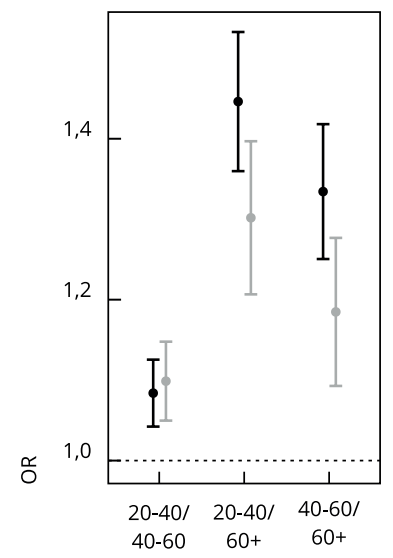

Contrastes [Faixa etária (anos)]

3g) 2014

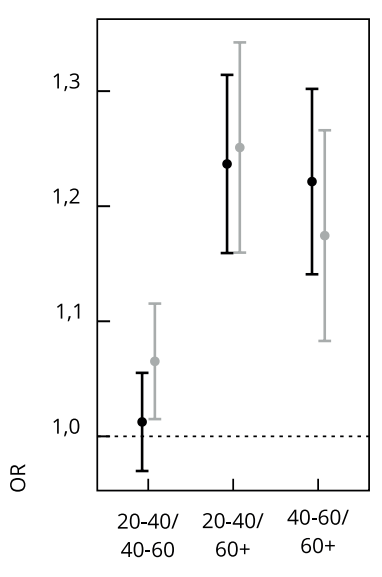

Contrastes [Faixa etária (anos)]

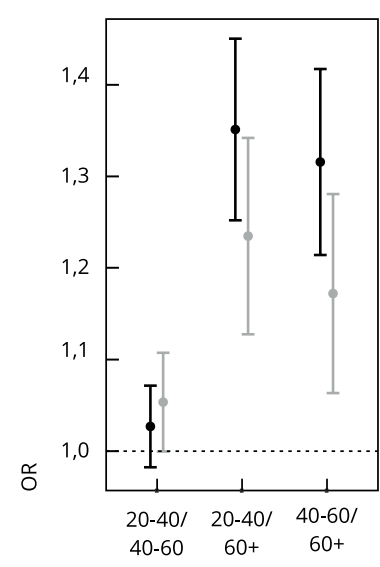

Contrastes [Faixa etária (anos)]

3h) 2015

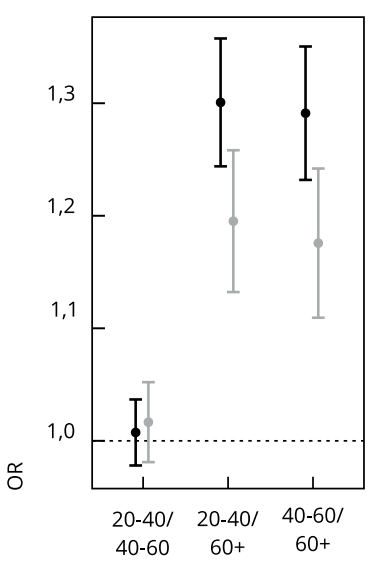

Contrastes [Faixa etária (anos)] 
O progredir da idade tem reflexos diretos na função cognitiva dos indivíduos, piorando a memória e a fluência verbal dos mais idosos. Essa situação é agravada em indivíduos de baixo nível escolar ${ }^{21}$. Assim, o decréscimo dos preenchimentos entre os idosos pode ter acontecido devido a uma piora da função cognitiva, resultando em uma menor chance em responder às perguntas realizadas pelo investigador. Nesse ponto, há também a hipótese de que o público mais jovem mereceria mais cuidado e atenção na visão do investigador, pois estes estariam mais atentos e ávidos a responder e questionar possíveis inconsistências do aplicador do questionário.

Os melhores preenchimentos no sexo feminino podem ter ocorrido devido, nos últimos anos, às mulheres terem tido políticas governamentais de saúde específicas, em detrimento dos homens, resultando em uma cultura de maior acesso e melhor uso dos serviços 22. Outra suposição é que as mulheres, por possuírem maior escolaridade, principalmente as coortes de nascimento mais recentes, estariam mais dispostas e esclarecidas a responder oportunamente ao questionamento sobre sua escolaridade. Os homens, principalmente os de menor escolaridade, estariam menos dispostos a responder por constrangimento ou incompreensão da pergunta realizada 23. Esse raciocínio também pode ser aplicado na explicação da relação entre a idade/sexo e o preenchimento. Segundo dados do Instituto de Pesquisa Econômica Aplicada (IPEA), as mulheres possuem maior escolaridade em todas as faixas etárias até os 60 anos, invertendo para uma maior escolaridade entre os homens a partir dessa idade 24. Isso explicaria o maior decréscimo relativo no preenchimento da escolaridade dos idosos entre as mulheres.

A semelhança dentre os preenchimentos de faixas de idade entre 20-40 anos e 40-60, deve-se provavelmente ao grau de exposição ser semelhante entre os pacientes destas faixas, que correspondem à idade laboral ativa da população. E assim ficariam expostos ao vetor do dengue de forma semelhante, repercutindo proporcionalmente nos indivíduos atendidos e notificados nos serviços de saúde.

Em 2015 e 2016, houve a introdução de outras arboviroses no cenário nacional, notadamente zika e chikungunya, que em aspectos clínicos são semelhantes ao dengue 25,26 . No entanto, devido à precocidade do entendimento desses eventos à época, esses agravos foram notificados no SINAN, erroneamente, como dengue, fato que levou a uma maior incidência de dengue nesses anos da série estudada. Como o número de casos aumentou bastante, a amostra ficou mais representativa e intensificou alguns achados como as diferenças entre os preenchimentos dos mais jovens comparativamente aos mais idosos terem acentuado nesses anos.

$\mathrm{O}$ dengue atinge em maior quantidade os mais pobres e estes possuem maior dificuldade de acesso aos sistemas de saúde 27,28,29. Um bom preenchimento da variável escolaridade, que refletiria as condições socioeconômicas, permitiria ter um vislumbre da subnotificação de casos de dengue entre os mais pobres, sendo, desta forma, ponto de partida para uma correção de dados de notificação. Pretendemos em um trabalho futuro desenvolver uma estratégia de correção para os casos de dengue que leve em consideração a escolaridade como indicador de acesso e notificação.

As principais limitações do estudo estão vinculadas à baixa qualidade dos dados obtidos de forma secundária. Possíveis casos de dengue não são notificados no sistema devido à falta de vigilância em saúde oportuna ou falta de acesso, variando em grau de local para local, levando a uma subnotificação de possíveis casos. Nesse ínterim do acesso, estudos apontam que os menos favorecidos são aqueles mais propensos a não buscar os serviços de saúde 27 , o que poderia levar a uma menor representatividade de casos notificados dentre os menos escolarizados. Por outro lado, este estudo considerou como desfecho o caso notificado, em vez do caso confirmado para dengue. Nesse caso, a população dos casos notificados estaria sobrenotificada pela presença de casos falso-positivos para dengue.

Os pontos positivos do estudo foram a utilização de uma base populacional, embora ressaltem-se as limitações descritas anteriormente, a especificação correta do modelo estatístico usado levando-se em conta a correlação intrínseca dos dados e a possível aplicabilidade da mesma metodologia para outras doenças, desde que não sejam doenças estritas a populações específicas como a infantil (coqueluche, por exemplo), o que restringiria a avaliação da escolaridade. 


\section{Conclusão}

Os sistemas de vigilância requerem avaliação contínua para que todos os seus dados resultem em interpretações precisas. O mau preenchimento das fichas favorece a geração de dados deficientes e duvidosos, o que contribui para o desconhecimento da magnitude da doença, desigualdade em saúde e definição de metas.

\section{Colaboradores}

L. M. Guimarães contribuiu com a concepção, projeto, análise e interpretação dos dados e redação do artigo e é responsável por todos os aspectos do trabalho na garantia da exatidão e integridade de qualquer parte da obra. G. M. Cunha contribuiu com a concepção, análise e interpretação dos dados, redação e revisão crítica do artigo e aprovou a versão final a ser publicada.

\section{Informações adicionais}

ORCID: Lucas Melo Guimarães (0000-0001-98016788); Geraldo Marcelo da Cunha (0000-00017128-933X).

\section{Agradecimentos}

Agradecemos ao Ministério da Saúde e à Fundação Oswaldo Cruz.

\section{Referências}

1. Departamento de Vigilância Epidemiológica, Secretaria de Vigilância em Saúde, Ministério da Saúde. Guia de vigilância epidemiológica. 6a Ed. Brasília: Ministério da Saúde; 2006. (Série A - Normas e Manuais Técnicos).

2. Malhão TA, Oliveira GP, Codennoti S, Moherdaui F. Avaliação da completitude do Sistema de Informação de Agravos de Notificação da Tuberculose, Brasil, 2001-2006. Epidemiol Serv Saúde 2010; 19:245-56.

3. Adler NE, Ostrove JM. Socioeconomic status and health: what we know and what we don't. Ann N Y Acad Sci 1999; 896:3-15.

4. Cockerham WC, organizador. The Blackwell companion to medical sociology. Oxford/Malden: Blackwell; 2001. (Blackwell Companions to Sociology).

5. Baker EH. Socioeconomic status, definition. In: Cockerham WC, Dingwall R, Quah S, organizadores. The Wiley Blackwell encyclopedia of health, illness, behavior, and society. Chichester: John Wiley \& Sons; 2014. p. 2210-4.

6. Hamid TA, Momtaz YA, Ibrahim R. Predictors and prevalence of successful aging among older Malaysians. Gerontology 2012; 58:366-70.

7. Instituto Brasileiro de Geografia e Estatística. Síntese de indicadores sociais: uma análise das condições de vida da população brasileira. Rio de Janeiro: Instituto Brasileiro de Geografia e Estatística; 2016.

8. Ministério da Saúde; Organização Pan-Ameicana da Saúde; Fundação Oswaldo Cruz. A experiência brasileira em sistemas de informação em saúde. Brasília: Editora MS; 2009.

9. Centro Nacional de Epidemiologia. Guia de vigilância epidemiológica. Brasília: Centro $\mathrm{Na}$ cional de Epidemiologia, Fundação Nacional de Saúde, Ministério da Saúde; 1998.

10. Ministério da Saúde. Guia de vigilância em saúde. 2a Ed. Brasília: Ministério da Saúde; 2017.

11. Silvestrin S, Buriol VCS, Silva CH, Goldani MZ. Avaliação da incompletude da variável escolaridade materna nos registros das Declarações de Nascidos Vivos nas capitais brasileiras - 1996 a 2013. Cad Saúde Pública 2018; 34:e00039217. 
12. Braz RM, Oliveira PTR, Reis AT, Machado NMS. Avaliação da completude da variável raça/cor nos sistemas nacionais de informação em saúde para aferição da equidade étnico-racial em indicadores usados pelo Índice de Desempenho do Sistema Único de Saúde. Saúde Debate 2013; 37:554-62.

13. Pedraza DF. Qualidade do Sistema de Informações sobre Nascidos Vivos (Sinasc): análise crítica da literatura. Ciênc Saúde Colet 2012; 17:2729-37

14. Santos NP, Lírio M, Passos LAR, Dias JP, Kritski AL, Galvão-Castro B, et al. Completeness of tuberculosis reporting forms in five Brazilian capitals with a high incidence of the disease. J Bras Pneumol 2013; 39:221-5.

15. Teresina. Lei Complementar $n^{\circ} 4.360$, de 22 de janeiro de 2013. Institui a Fundação Hospitalar de Teresina - FHT, e dá outras providências. Diário Oficial do Município 2013; 22 jan.

16. Costa JMBS, Frias PG. Avaliação da completitude das variáveis da Declaração de Nascido Vivo de residentes em Pernambuco, Brasil, 1996 a 2005. Cad Saúde Pública 2009; 25: 613-24.

17. Guerra FAR, Llerena Jr. JC, Gama SGN, Cunha $\mathrm{CB}$, Theme Filha MM. Confiabilidade das informações das declarações de nascido vivo com registro de defeitos congênitos no Município do Rio de Janeiro, Brasil, 2004. Cad Saúde Pública 2008; 24:438-46.

18. Jorge MHPM, Laurenti R, Gotlieb SLD. Análise da qualidade das estatísticas vitais brasileiras: a experiência de implantação do SIM e do SINASC. Ciênc Saúde Colet 2007; 12:643-54.

19. Mascarenhas MDM, Gomes KRO. Confiabilidade dos dados do Sistema de Informações sobre Nascidos Vivos em Teresina, Estado do Piauí, Brasil - 2002. Ciênc Saúde Colet 2011; 16 Suppl 1:1233-9.

20. Krieger N, Williams DR, Moss NE. Measuring social class in US public health research: concepts, methodologies, and guidelines. Annu Rev Public Health 1997; 18:341-78.
21. Castro-Costa E, Lima-Costa MF, Andrade FB, Souza Junior PRB, Ferri CP. Cognitive function among older adults. Rev Saúde Pública 2019; 52 Suppl 2:4s.

22. Ministério da Saúde. Política Nacional de Atenção Integral à Saúde da Mulher: princípios e diretrizes. Brasília: Editora MS; 2004. (Série C - Projetos, Programas e Relatórios).

23. Peret E. Mulher estuda mais, trabalha mais e ganha menos do que o homem. Agência IBGE Notícias 2018; 7 mar. https://agenciadenoti cias.ibge.gov.br/agencia-noticias/2012-agen cia-de-noticias/noticias/20234-mulher-estu da-mais-trabalha-mais-e-ganha-menos-doque-o-homem.

24. Instituto de Pesquisa Econômica Aplicada. Retrato das desigualdades de gênero e raça. http://www.ipea.gov.br/retrato/indicadores_ educacao.html (acessado em 10/Jun/2019).

25. Lang P. Zika, chikungunya e dengue: entenda as diferenças. Agência Fiocruz de Notícias 2015; 17 nov. https://agencia.fiocruz.br/zikachikungunya-e-dengue-entenda-diferen $\%$ C3\%A7as.

26. Nunes ML, Carlini CR, Marinowic D, Kalil Neto F, Fiori HH, Scotta MC, et al. Microcephaly and Zika virus: a clinical and epidemiological analysis of the current outbreak in Brazil. J Pediatr (Rio J.) 2016; 92:230-40.

27. Barata RB. Como e por que as desigualdades sociais fazem mal à saúde. Rio de Janeiro: Editora Fiocruz; 2009.

28. Burattini MN, Lopez LF, Coutinho FAB, Siqueira JB, Homsani S, Sarti E, et al. Age and regional differences in clinical presentation and risk of hospitalization for dengue in Brazil, 2000-2014. Clinics 2016; 71:455-63.

29. Johansen IC, Carmo RL, Alves LC, Dias Bueno MDC. Environmental and demographic determinants of dengue incidence in Brazil. Rev Salud Pública 2018; 20:346-53. 


\section{Abstract}

Brazil is among the countries of the world with the most dengue cases. Case reporting systems are essential for monitoring the disease's evolution, but few reporting forms are properly completed. Knowledge of the patients' schooling level is important for understanding the role of social inequality in the risk of illness. The article aims to describe the completion of the schooling item on dengue notification forms provided by Brazilian Information System on Diseases of Notification (SINAN) from 2008 to 2017, according to sex and age bracket in the state capitals of the Northeast and Southeast regions. The target outcome was the completion of the schooling item on the reporting form. The predicted probabilities of completion of the schooling item were obtained with multilevel logistic models. Completion was low in the state capitals (less than $30 \%$ in eight of them). The odds of having the schooling item completed decreased with age, independently of the patient's sex and the reporting year. Women up to 60 years showed higher odds than men of completion of the schooling item. Overall, men showed $9 \%$ lower odds of having the schooling item completed, compared to women. The model with interaction showed an increase in the difference, that is, the completion rate decreased with advancing age, especially among women. Given the above, incomplete dengue reporting forms lead to insufficient and dubious data. Although schooling is not fully appreciated at the moment of completing the reporting form, it is essential information in the context of dengue surveillance.

Dengue; Health Information Systems; Educational Status

\section{Resumen}

Brasil figura entre los países con mayor número de casos de dengue en el mundo. Los sistemas de notificación son fundamentales para acompañar la evolución de la enfermedad, no obstante, se muestran con lagunas por la falta de cumplimentación. El conocimiento del nivel educacional de los individuos es importante para la compresión del papel de la desigualdad social en el riesgo de enfermedad. El objetivo de este artículo es describir la cumplimentación de la escolaridad en las fichas de notificación sobre el dengue, puestas a disposición del Sistema Brasileño de Información de Enfermedades de Notificación (SINAN) entre 2008 y 2017, por sexo y franja de edad, entre las capitales de las regiones Nordeste y Sudeste del pais. El resultado de interés fue la cumplimentación de la variable escolaridad. Las probabilidades predichas de cumplimentación de la escolaridad se obtuvieron utilizándose modelos logísticos multinivel. La cumplimentación de la escolaridad fue baja en las capitales, siendo en ocho de ellas, inferior a un 30\% durante el periodo. La oportunidad de contar con la escolaridad completa decreció cuanto más avanzaba la edad, independientemente del sexo y del año. Las mujeres de hasta 60 años tuvieron mayores oportunidades de cumplimentar la escolaridad que los hombres. En general, los hombres tuvieron un $9 \%$ menos oportunidad de contar con la escolaridad cumplimentada que las mujeres. En el modelo con interacción hubo una intensificación de la diferencia, o sea, existió un empeoramiento -respecto a la cumplimentación de la escolaridad- a medida que avanzaba la edad, principalmente entre mujeres. Ante lo expuesto, la mala cumplimentación de las fichas favorece la generación de datos deficientes y dudosos, y la escolaridad, aunque poco valorada en el momento de rellenar los datos, tiene una importancia central en esta coyuntura.

Dengue; Sistemas de Información en Salud; Escolaridad
Recebido em 30/Set/2019

Versão final reapresentada em 06/Fev/2020 Aprovado em 01/Abr/2020 